\title{
Self-expandable transcatheter aortic valve for surgical prosthetic aortic valve dysfunction
}

\author{
Michael Ibrahim ${ }^{1,2}$, Joshua Grimm ${ }^{1}$, Matthew Woods ${ }^{1}$, Paul N. Fiorilli ${ }^{3}$, Wilson Y. Szeto ${ }^{1}$ \\ ${ }^{1}$ Division of Cardiovascular Surgery, The University of Pennsylvania, Philadelphia, PA, USA; ${ }^{2}$ Penn Cardiovascular Institute, The University of \\ Pennsylvania, Philadelphia, PA, USA; ${ }^{3}$ Division of Cardiology, The University of Pennsylvania, Philadelphia, PA, USA \\ Correspondence to: Wilson Y. Szeto, MD. Professor of Surgery, University of Pennsylvania School of Medicine, Vice Chief of Clinical \\ Operations and Quality, Division of Cardiovascular Surgery, Chief, Cardiovascular Surgery at Penn Presbyterian Medical Center, Surgical \\ Director, Transcatheter Cardio-Aortic, Therapies 51 North 39th Street, Heart and Vascular Pavilion Suite 2A, Philadelphia, PA 19104, USA. \\ Email: wilson.szeto@pennmedicine.upenn.edu.
}

Submitted Apr 27, 2021. Accepted for publication Jul 14, 2021.

doi: 10.21037/acs-2021-tviv-16

View this article at: https://dx.doi.org/10.21037/acs-2021-tviv-16

As the prevalence of surgical bioprosthetic aortic valve replacement (SVAR) increases (1), more patients will likely suffer from structural valvular deterioration (SVD). Repeat SAVR (R-SAVR) has been the standard of care and has several advantages, including the ability to replace a bioprosthetic valve with any other valve required, the ability to enlarge the annulus if needed and the ability to directly confirm coronary clearance. Likewise, valve-in-valve transcatheter aortic valve replacement (ViV-TAVR) has advantages, including the avoidance of repeat sternotomy, which has risks of mortality and complications, and shorter hospitalization. However, ViV-TAVR is associated with the risk of coronary obstruction and unknown valve durability. Taken together, there are short-term considerations such as safety, coronary obstruction, stroke, procedural success without para-valvular leak and freedom from coronary obstruction for ViV-TAVR. Long-term considerations include long-term survival, hemodynamics, durability and the facility of future valvular interventions. There is no direct randomized data which compares R-SAVR to ViVTAVR. However, several metanalyses have shed light on both short-term and longer-term issues.

\section{Survival}

Pompeu et al. recently published a meta-analysis of twelve studies including a combined 16,207 patients undergoing R-SAVR or ViV-TAVR (2). This and other studies (3) suggest that thirty-day mortality is lower with ViV-TAVR than R-SAVR. This mirrors the findings of transcatheter versus surgical trials, where less invasive procedures have lower early mortality. Again, mirroring native valve studies, this early advantage is diminished over time, with one-year mortality being no different in the latest meta-analysis (2). Patients in these studies tend to be in their late-seventies, with up to $25 \%$ mortality at three years for ViV-TAVR patients. Future studies must examine long-term mortality, especially if they include, or intend to be used to guide treatment in younger patients with longer life expectancy.

ViV-TAVR appeared to be associated with a lower rate of complications compared to R-SAVR, including a lower rate of major bleeding and shorter hospitalization (2). Stroke rate appeared to be equivalent between both modalities (2), or occurred too infrequently to be compared (3). Coronary occlusion is a particularly feared complication of ViVTAVR; the rates of coronary occlusion appeared to be similar between balloon and self-expandable TAVR.

\section{Patient prosthesis mismatch}

Patient prosthesis mismatch (PPM) appeared to be one of the major limitations of ViV-TAVR, especially when there is a small bioprosthesis (2). Up to $35 \%$ of $\mathrm{ViV}$ TAVR patients suffered severe PPM (4). In a study of over 1,000 ViV-TAVRs, small prostheses were associated with late mortality at eight years (5). The rate of severe PPM appeared to be significantly increased after ViV-TAVR compared to R-SAVR (2), which has been associated with late mortality (6). PPM after ViV-TAVR is less of an issue with larger bioprostheses, and may be partially alleviated by 
valve fracture or the use of prostheses designed for TAVR implantation with expandable sewing rings. Valve fracture involves inflation of a high-pressure balloon across the rigid annuloplasty ring until the "waist" in the balloon disappears, often heralded by an audible cracking noise. This results in significant improvement in valve gradients and allows implantation of a larger TAVR valve (7). Self-expanding valves sit in the supra-annular position and are therefore not constrained by the annulus. In general, we favor selfexpandable supra-annular valves with valve fracture as needed for small bioprostheses.

\section{Durability}

Available data suggests that ViV-TAVR durability may be limited, with $10 \%$ of valves suffering SVD at three years $(4,5)$. In particular, balloon-expandable valves appeared to be associated with higher incidence of post ViV-TAVR re-intervention (5). As experience grows, the nuances of which TAVR valve is most suitable to implant and in which specific bioprosthesis will mature. Data from exvivo experiments show that the optimal implantation height associated with the best hemodynamic profile varies for each valve and TAVR combination (8). Using a physiologic left heart simulator, this group demonstrated that selfexpandable valves had optimal hemodynamics at the normal implantation depth, whereas balloon expandable valves had their best hemodynamic profile at a +6 supraannular position, when tested in a Perimount prosthesis (8). Indeed, self-expandable valves had better $\mathrm{ViV}$ performance than balloon-expandable valves under these conditions. The impact of this on durability is a matter of active investigation.

\section{Decision-making}

Repeat valve interventions require careful decision-making, with input from surgeons and cardiologists together as a heart team. The center of this team must be the patient, whose individual anatomy, clinical status and values will inform a shared decision-making process. This approach is rightly associated with a class I recommendation in valvular heart disease management guidelines (9). The same set of factors which determine pre-procedural decisions also dominate our response to residual gradients and leaks, with a high bar for well-functioning patients with an extended life expectancy. Although the concept of a SAVR followed by a TAVR has gained traction, we must be careful to caution patients about the unknown impact of this strategy on valve durability and survival, as data is still accumulating. Valve performance is another important concept for these patients, whose active lifestyles may be better served by a Ross operation, or in some limited cases, by mechanical valve implantation.

\section{Conclusions}

Most studies have shown largely equivalent outcomes between ViV-TAVR and R-SAVR, especially with respect to early mortality $(2,5,10)$. On the one hand, this is an impressive result for ViV-TAVR since this cohort is typically higher risk than patients undergoing R-SAVR. On the other hand, these data are the result of optimized patient selection for either modality. It is clear that a randomized trial is needed and will occur in the near future. These trials must clarify several important questions. First, are short term outcomes equivalent between ViV-TAVR and R-SAVR, and how do these compare in the medium and long-term? Second, what is the long-term durability of ViV-TAVR? Third, which patient cohorts benefit from each therapy as a primary strategy, and with which TAVR valves and in which bioprostheses? Fourth, what are the serial hemodynamic performance profiles of these valves? Until randomized data is available, we should continue to select patients who stand to benefit from either therapy based on their individual characteristics and alongside their predicted risk profiles and life expectancy.

\section{Acknowledgments}

Funding: None.

\section{Footnote}

Conflicts of Interest: PF: Edwards Lifesciences: Investigator, speaker; Medtronic: Investigator, speaker. WYS: Edwards Lifesciences: investigator, advisory board, speaker; Medtronic: investigator, advisory board, speaker. The other authors have no conflicts of interest to declare.

Open Access Statement: This is an Open Access article distributed in accordance with the Creative Commons Attribution-NonCommercial-NoDerivs 4.0 International License (CC BY-NC-ND 4.0), which permits the noncommercial replication and distribution of the article with the strict proviso that no changes or edits are made and the 
original work is properly cited (including links to both the formal publication through the relevant DOI and the license). See: https://creativecommons.org/licenses/by-nc-nd/4.0/.

\section{References}

1. Goldstone $\mathrm{AB}$, Chiu $\mathrm{P}$, Baiocchi $\mathrm{M}$, et al. Mechanical or Biologic Prostheses for Aortic-Valve and Mitral-Valve Replacement. N Engl J Med 2017;377:1847-57.

2. Sá MPBO, Van den Eynde J, Simonato M, et al. Valvein-Valve Transcatheter Aortic Valve Replacement Versus Redo Surgical Aortic Valve Replacement: An Updated Meta-Analysis. JACC Cardiovasc Interv 2021;14:211-20.

3. Malik AH, Yandrapalli S, Zaid S, et al. Valve-in-Valve Transcatheter Implantation Versus Redo Surgical Aortic Valve Replacement. Am J Cardiol 2020;125:1378-84.

4. de Freitas Campos Guimarães L, Urena M, Wijeysundera HC, et al. Long-Term Outcomes After Transcatheter Aortic Valve-in-Valve Replacement. Circ Cardiovasc Interv 2018;11:e007038.

5. Bleiziffer S, Simonato M, Webb JG, et al. Long-term

Cite this article as: Ibrahim M, Grimm J, Woods M, Fiorilli PN, Szeto WY. Self-expandable transcatheter aortic valve for surgical prosthetic aortic valve dysfunction. Ann Cardiothorac Surg 2021;10(5):689-691. doi: 10.21037/acs-2021-tviv-16 outcomes after transcatheter aortic valve implantation in failed bioprosthetic valves. Eur Heart J 2020;41:2731-42.

6. Elmahdy W, Osman M, Farag M, et al. Prosthesis-Patient Mismatch Increases Early and Late Mortality in Low Risk Aortic Valve Replacement. Semin Thorac Cardiovasc Surg 2021;33:23-30.

7. Chhatriwalla AK, Allen KB, Saxon JT, et al. Bioprosthetic Valve Fracture Improves the Hemodynamic Results of Valve-in-Valve Transcatheter Aortic Valve Replacement. Circ Cardiovasc Interv 2017;10:e005216.

8. Midha PA, Raghav V, Condado JF, et al. Valve Type, Size, and Deployment Location Affect Hemodynamics in an In Vitro Valve-in-Valve Model. JACC Cardiovasc Interv 2016;9:1618-28.

9. Falk V, Baumgartner H, Bax JJ, et al. 2017 ESC/EACTS Guidelines for the management of valvular heart disease. Eur J Cardiothorac Surg 2017;52:616-64.

10. Neupane S, Singh H, Lämmer J, et al. Meta-Analysis of Transcatheter Valve-in-Valve Implantation Versus Redo Aortic Valve Surgery for Bioprosthetic Aortic Valve Dysfunction. Am J Cardiol 2018;121:1593-600. 